\title{
A Novel Application of Zero-Current-Switching Quasiresonant Buck Converter for Battery Chargers
}

\author{
Kuo-Kuang Chen \\ Department of Electrical Engineering, Far East University, Tainan city 744, Taiwan \\ Correspondence should be addressed to Kuo-Kuang Chen, chenkk@cc.feu.edu.tw
}

Received 24 April 2011; Accepted 27 May 2011

Academic Editor: Xing-Gang Yan

Copyright (C) 2011 Kuo-Kuang Chen. This is an open access article distributed under the Creative Commons Attribution License, which permits unrestricted use, distribution, and reproduction in any medium, provided the original work is properly cited.

The main purpose of this paper is to develop a novel application of a resonant switch converter for battery chargers. A zero-current-switching (ZCS) converter with a quasiresonant converter (QRC) was used as the main structure. The proposed ZCS dc-dc battery charger has a straightforward structure, low cost, easy control, and high efficiency. The operating principles and design procedure of the proposed charger are thoroughly analyzed. The optimal values of the resonant components are computed by applying the characteristic curve and electric functions derived from the circuit configuration. Experiments were conducted using lead-acid batteries. The optimal parameters of the resonance components were determined using the load characteristic curve diagrams. These values enable the battery charger to turn on and off at zero current, resulting in a reduction of switching losses. The results of the experiments show that when compared with the traditional pulse-width-modulation (PWM) converter for a battery charger, the buck converter with a zero- current-switching quasiresonant converter can lower the temperature of the activepower switch.

\section{Introduction}

Batteries are extensively utilized in many applications, including renewable energy generation systems, electrical vehicles, uninterruptible power supplies, laptop computers, personal digital assistants, cell phones, and digital cameras. Since these appliances continuously consume electric energy, they need charging circuits for batteries. Efficient charging shortens the charging time and extends the battery service life, while harmless charging prolongs the battery cycle life and achieves a low battery operating cost. Moreover, the charging time and lifetime of the battery depend strongly on the properties of the charger circuit. The development of battery chargers is important for these devices. A good charging method can enhance battery efficiency, prolong battery life, and improve charge speed. Several charging circuits have been proposed to overcome the disadvantages of the traditional battery charger. 


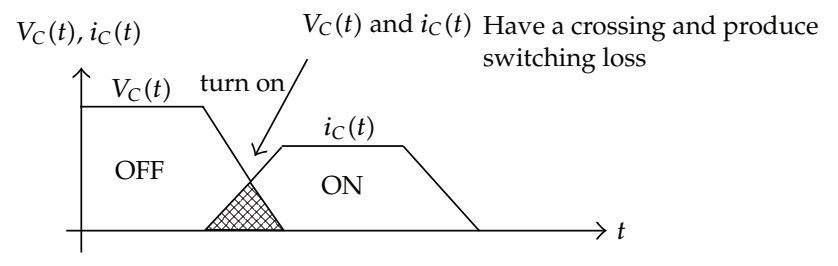

Figure 1: The switching loss of a traditional PWM power transistor.

The linear power supply is the simplest. A $60-\mathrm{Hz}$ transformer is required to deliver the output within the desired voltage range. However, the linear power supply is operated at the line frequency, which makes it large both in size and weight. Besides, the system conversion efficiency is low because the transistor operates in the active region. Hence, when higher power is required, the use of an overweighted and oversized line-frequency transformer makes this approach impractical. The high-frequency operation of the conventional converter topologies depends on a considerable reduction in switching losses to minimize size and weight. Many soft-switching techniques have been proposed in recent years to solve these problems. Neti R. M. Rao developed the traditional pulse width modulation (PWM) power converter in 1970. PWM was used to control the turn-on-time of the power transistors to achieve the target of voltage step-up and step-down. The switching loss of traditional PWM converters is shown in Figure 1, where $V_{C}(t)$ is the voltage across both the collector and emitter of the transistor, and $i_{C}(t)$ is the current across the collector of the transistor.

The advantages and drawbacks of this modulation style are addressed as follows. Advantages

(1) A high switching frequency can reduce the volume of magnetic elements and capacitors.

(2) Power transistors are operated in the saturation region and cut-off region. This makes the power loss of the power transistors nearly zero.

Drawbacks

(1) The power is still restrained voltage and current during switching period, resulting in switching losses.

(2) Fast switching can result in serious spike current $(d i / d t)$, voltage $(d v / d t)$, and electromagnetic interference (EMI).

The control switches in all the PWM dc-dc converter topologies operate in a switch mode, in which they turn a whole load current on and off during each switching. This switch-mode operation subjects the control switches to high switching stress and high switching power losses. To maximize the performance of switch-mode power electronic conversion systems, the switching frequency of the power semiconductor devices needs to be increased, but this results in increased switching losses and electromagnetic interference. To eradicate these problems, soft switching and various charger topologies more suitable for battery energy storage systems have been presented and investigated. Zero-voltageswitching (ZVS) and zero-current-switching (ZCS) techniques are two conventionally employed soft-switching methods. These techniques lead to either zero voltage or zero current during switching transition, significantly decreasing the switching losses and 


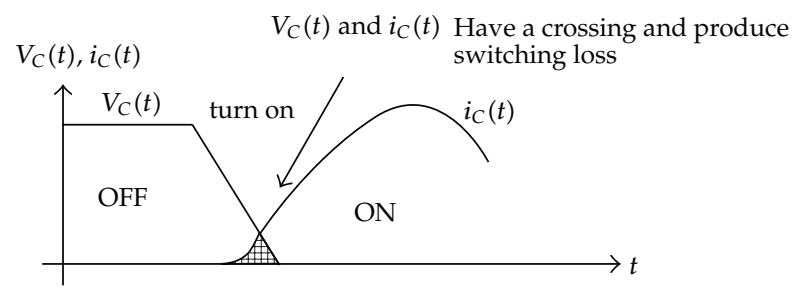

Figure 2: The switching loss of resonant power transistor.

increasing the reliability for the battery chargers. The ZVS technique eliminates capacitive turn-on losses and decreases the turnoff switching losses by slowing down the voltage rise, thereby lowering the overlap between the switch voltage and the switch current. However, a large external resonant capacitor is needed to lower the turnoff switching loss effectively for ZVS. Conversely, ZCS eliminates the voltage and current overlap by forcing the switch current to zero before the switch voltage rises, making it more effective than ZVS in reducing switching losses, particularly for slow switching power devices. For high-efficiency power conversion, the ZCS topologies are most frequently adopted.

This paper adopts zero-current-switching (ZCS) converter with quasiresonant converter (QRC) as the main structure to charge a lead-acid battery. The resonant phenomena of ZCS converter with QRC is used to determine the switching loss of the switch. Traditional PWM power converters have nonideal power loss during switching procedure. A capacitor in parallel with the switch is adopted in the proposed structure. Both the inductor and capacitor resonate to make the current into sine waves. This can reduce the overlap area of the voltage and current waves, decreasing switching loss. The switching loss of a resonant power converter is shown in Figure 2.

In the attempt to overcome the tradition PWM converters, many efforts have been made to search a less expensive charger topology for batteries to offer a competitive price in the consumer market. This paper presents a relatively simple topology for the battery charger with a ZCS quasiresonant buck converter, which is the most economical circuit topology commonly used for driving low power energy storage systems. In the proposed approach, a resonant tank is interposed between the input dc source and the battery. With the added resonant tank, the battery charger can achieve low switching loss with only one additional active power switch and easy control circuitry.

Power converters can be divided into the following types [1-8].

(1) Resonant Converter $(R C)$ : this converter uses both the half-bridge circuit and the fullbridge circuit as basic structures. It is implemented as a series resonant converter or parallel resonant converter.

(2) Quasiresonant Converter $(Q R C)$ : this converter adopts both the half-wave style circuit and the full-wave style circuit as basic structures. It is implemented as a ZCS converter or zero voltage switching (ZVS) converter.

(3) Multiresonant Converter (MRC): this converter adopts both a ZCS circuit and a partial resonant ZVS circuit in the half-bridge style DC-DC converter as basic structures.

The advantages and drawbacks of resonant power converters are given below.

Advantages: the power transistor has no voltage or current during the switching process. It can reduce switching loss and restrain EMI effectively. 


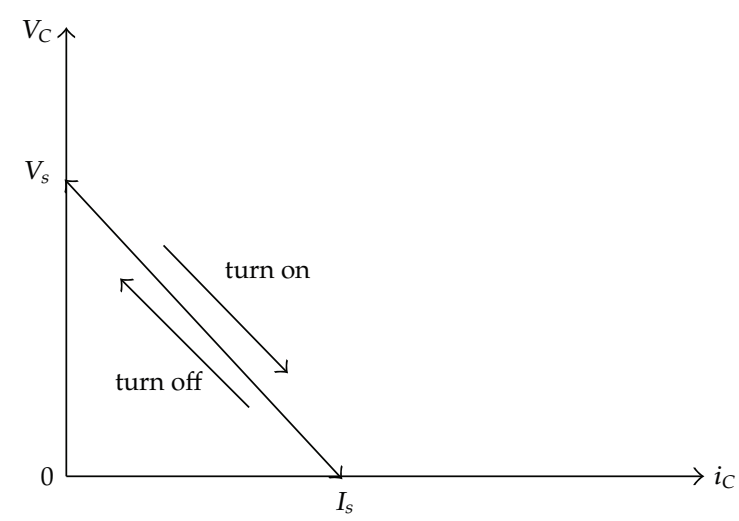

Figure 3: The dimensions of hard switching.

Drawbacks: the resonance technique increases the voltage and current stress on components. A parasitized capacitor can result in serious conduction loss.

Let $V_{C}(t)$ and $i_{C}(t)$ turn on and cut off at the same time, as shown in Figure 3. Assuming that their variation time is $\Delta t$. The initial turn-on time is adopted from

$$
\begin{gathered}
V_{C}(t)=-\frac{V_{S}}{\Delta t} t+V_{S}, \\
i_{C}(t)=\frac{I_{S}}{\Delta t} t,
\end{gathered}
$$

where $V_{s}$ is the voltage of both the collector and emitter in the transistor during the turn-off period, and $I_{S}$ is the collector current during the turn-on period. The switching loss can be written as

$$
P_{C}(t)=V_{C}(t) i_{C}(t)
$$

\section{The Investigation of a Lead-Acid Secondary Battery}

Batteries have become an increasingly important energy source. They can convert electrical energy into physical energy. Batteries can be divided into physical energy and chemical energy types. Physical type batteries convert both solar energy and thermal energy into electrical energy using physical energy. Using the oxidation-reduction reactions of electrochemistry is currently very popular. The chemical energy of active materials is converted into electrical energy in chemical energy batteries. All batteries contain energy produced chemical electrolysis. Normal batteries use electrolysis only. If we add extra energy into the battery, the battery stored the energy by antireaction. The battery releases energy by way of electrolysis. Lead-acid batteries are traditional energy-storage devices. They have a large electromotive force (EMF) and a wide of range operation temperature. Their advantages are a simple structure, mature technology, cheap price, and excellent cycle life. For the above reasons, lead-acid batteries are still important today. This paper uses a lead-acid battery as the load for the charging test. A lead-acid second battery made by Man-Shiung Corporation was chosen. When a lead-acid battery is connected to a load, the interior reaction of the lead-acid 


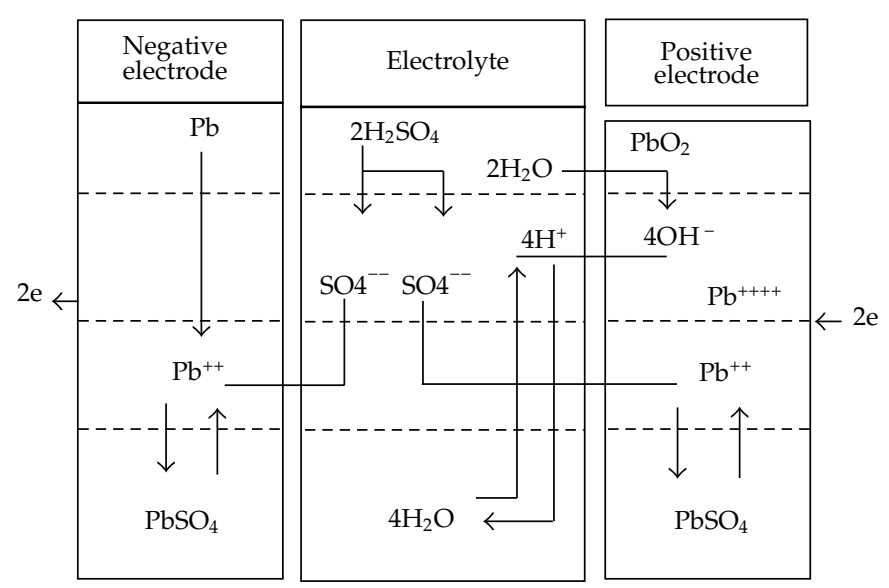

Figure 4: Discharge reaction.

battery is the discharge reaction. The chemical reaction is described in [9]

$$
\mathrm{PbO}_{2}+\mathrm{Pb}^{2+}+2 \mathrm{H}_{2} \mathrm{SO}_{4}+2 \mathrm{e}^{-} \Longrightarrow 2 \mathrm{PbSO}_{4}+2 \mathrm{H}_{2} \mathrm{O} \text {. }
$$

As shown in Figure 4, lead-acid batteries produce both lead sulfate and water during the discharge period. At the positive electrode, lead dioxide reacts with sulfuric acid in the electrolyte during the discharge period. Sulfuric acid is decomposed at the electrode. Sulfuric acid reacts with lead dioxide, which is the activated material at the positive electrode. This reaction produces lead sulfate, which sinks and piles up at the electrode. Lead, the activated material, reacts with sulfuric acid in the electrolyte at the negative electrode. Lead sulfate is produced from the above reaction. Then, lead sulfate sinks and piles up at the electrode. In the electrolyte, sulfuric acid is decomposed by reactions with the activated material at both the positive and negative electrodes. The reaction reduces the electrolyte concentration. A lot of lead sulfate sinks and piles up at both the positive and negative electrodes. This reaction increases the interior resistance of the battery and decreases the voltage of the lead acid battery. As shown in Figure 5, the lead-acid battery recharges when it is discharged to a certain level. The interior reaction of the battery is the charging reaction, as shown in

$$
2 \mathrm{PbSO}_{4}+2 \mathrm{H}_{2} \mathrm{O} \Longrightarrow \mathrm{PbO}_{2}+2 \mathrm{H}_{2} \mathrm{SO}_{4}+\mathrm{Pb}
$$

At both the positive and negative electrodes, the charge electrical energy of the exterior supply produces lead sulfate that is needed during the discharge period. After the above reaction, the activated material, lead dioxide, is deposed at the positive electrode. Lead reacts with sulfuric acid in the electrolyte at the negative electrode at same time. Their reactions increase the electrolyte concentration and raise voltage. In a lead-acid battery, the electrochemistry reactions of both charging and discharging are reversible. This is the socalled "Double Sulfate Theory." It can be expressed as

$$
\mathrm{PbO}_{2}+2 \mathrm{H}_{2} \mathrm{SO}_{4}+\mathrm{Pb} \Longleftrightarrow 2 \mathrm{PbSO}_{4}+2 \mathrm{H}_{2} \mathrm{O}
$$

The water production in lead-acid batteries during the discharge period is reelectrolysis by means of the charging reaction. From the above reactions, oxide is produced at the 


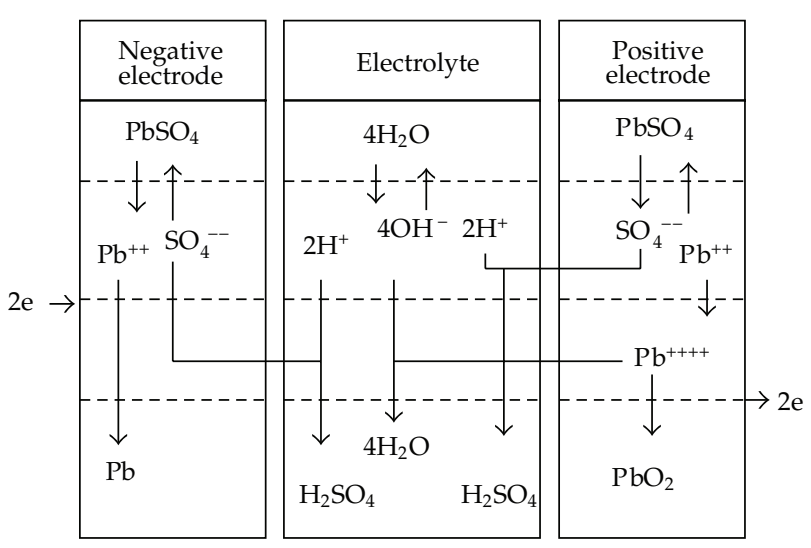

Figure 5: Charge reaction.

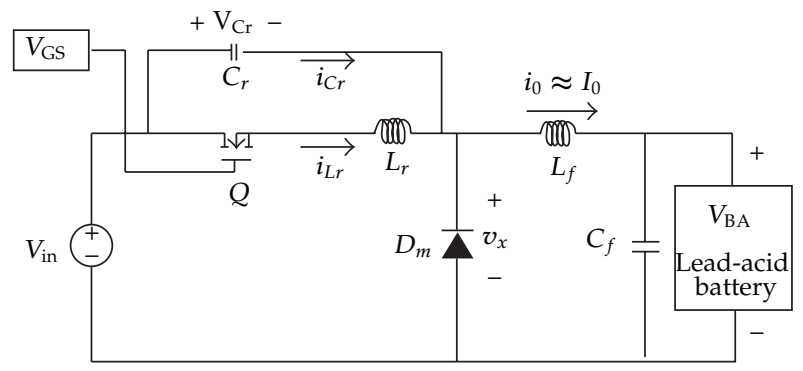

Figure 6: The ZCS QRC charger.

positive electrode, and hydrogen is produced at the negative electrode at the same time. This prevents water loss from the electrolyte in a closed lead-acid battery. Oxide is produced from the positive electrode during the charging period; the activated material, that is, lead, is obtained from the negative electrode. The two materials above react to make lead monooxide. Lead monooxide reacts with the sulfuric acid of lead sulfuric acid. Oxide produced from the positive electrode during the charging period is absorbed by the negative electrode. The oxide does not leave from the battery, resulting in water loss in the electrolyte.

In order to charge a battery properly, four charge modes should be designed and implemented in sequence, which are trickle charge, bulk charge, overcharge, and float charge. At the beginning of charge process, the trickle charge mode is adopted. And a very low constant current is applied to the battery to raise the voltage to the deep discharge threshold. Then the mode is switched into bulk charge. At the stage, a constant current is applied to the battery with the purpose of quickly replenishing electricity to the battery. When the voltage of battery exceeds overcharge limits, it enters into overcharge mode. In this mode a constant voltage is applied to the battery, and its value is typically set between $2.45 \mathrm{~V} /$ cell and $2.65 \mathrm{~V} /$ cell. Float charge is also a constant voltage charge mode after completing charge process to maintain the capacity of the battery against self-discharge.

\section{ZCS-QRC Buck Converter for a Battery Charger}

A variety of driving circuits have been employed for the ZCS quasiresonant buck converter. Conventionally, the trigger signal is associated with a proper duty cycle to drive the 


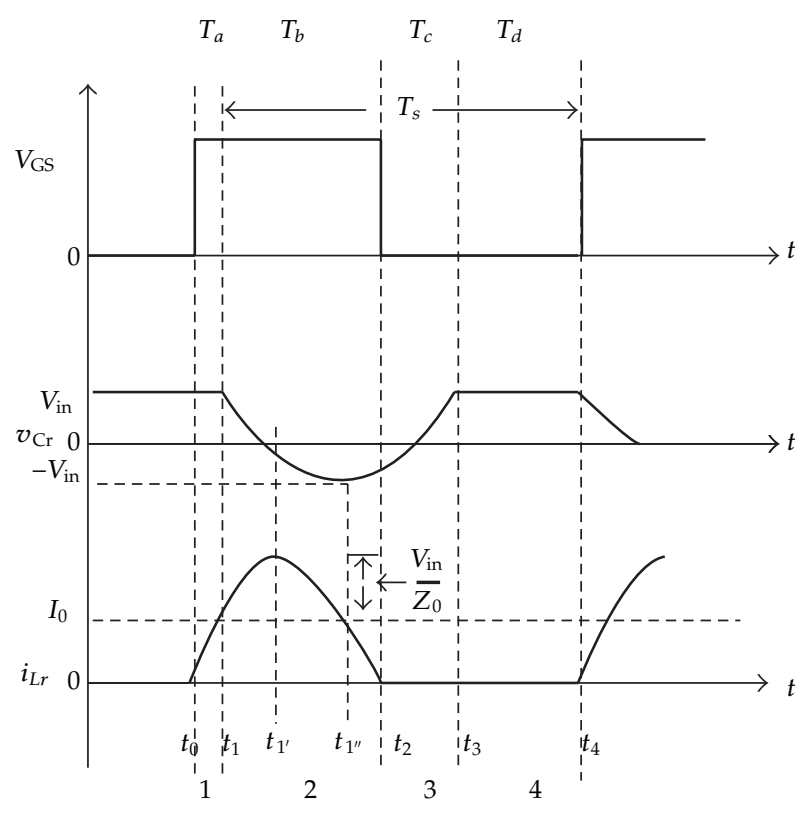

Figure 7: Timing and waveform diagram.

active power switch with the required charging current. The major elements of the ZCS quasiresonant buck converter for battery charger are available in a single integrated circuit. The integrated circuit contains an error amplifier, sawtooth waveform generator, and comparator for PWM. The turn-on and turn-off of the ZCS-QRC switch is operated when the current is zero. The produced current that is resonated by $L_{r}$ and $C_{r}$ passes through the switch. Because $L_{r}$ is very large, $i_{o}$ is assumed to be a constant $I_{o}$. The circuit structure is shown in Figure 6. In addition, the steady-state waveform is shown in Figure 7.

The following assumptions are made

(1) All semiconductor elements are ideal. This means that switches have no time delay during the switching period.

(2) There is no forward voltage drop in the diode $D_{m}$ during the turn-on period. There is no leakage current during the turn-off period.

(3) The inductor and capacitor of the tank circuit have no equivalent series resistance (ESR).

(4) The filtering inductor $L_{f} \gg L_{r}$ the filtering capacitor $C_{f} \gg C_{r}$. Because the cut-off frequency of current that is composed of low pass filter circuit load and filtering capacitor $C_{r}$ is much less than the resonant phase angle frequency $\omega_{o}=1 / \sqrt{L_{r} C_{r}}$ of resonant circuit that is composed by resonant inductor $L_{r}$ and resonant capacitor $C_{r}$. Compared to the resonant circuit, the filtering circuit composed of $L_{f}$ and $C_{f}$ and the load can be regarded as a constant current source $I_{o}$.

(5) Unregulated line voltage $V_{\text {in }}$ does not significantly vary during the resonant circuit turn-on and turn-off period $T_{s} . V_{\text {in }}$ is regarded as a constant.

The operation of the complete circuit is divided into four modes. 


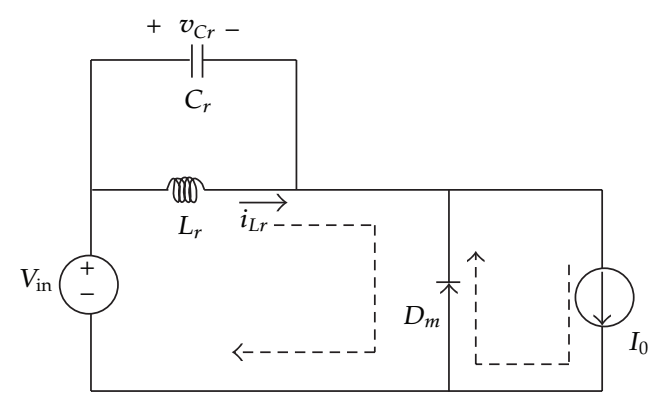

Figure 8: The equivalent circuit of Mode 1.

Mode 1 [linear stage $\left(t_{0} \leq t \leq t_{1}\right)$ ]

Before turning on the switch, the output current $I_{o}$ passes through diode $D_{m}$. Thus, the voltage across $C_{r}$ makes $v_{C r}=V_{i n}$. Thus, the initial conditions are $i_{L r}=0$ and $v_{C r}=V_{i n}$. The current that passes through the switch is zero at $t=t_{0}$. The switch $Q$ is turned on at the same time $t=t_{0}$ with ZCS. Diode $D_{m}$ is also turned on simultaneously. The inductor current $i_{L r}(t)$ is increased linearly. If $i_{L r}$ is less than $I_{o}$, The freewheel diode $D_{m}$ is still turned on, and $v_{C r}$ is maintained at $V_{i n}$, as shown in Figure 8. The circuit equation is represented as

$$
i_{L r}(t)=\frac{V_{\text {in }}}{L_{r}} t .
$$

This mode is finished when $i_{L r}(t)$ is equal to $I_{o}$ at $t=t_{1}$. The period of Mode 1 can be calculated by

$$
T_{a}=\frac{I_{o} L_{r}}{V_{\text {in }}}
$$

At $t=t_{1}$, diode $D_{m}$ is turned off. Then, the mode enters to Mode 2 .

Mode 2 [resonant stage $t_{1} \leq t \leq t_{2}$ ]

$L_{r}$ and $C_{r}$ resonate at this stage. The peak value of $i_{L r}$ is $V_{i n} / Z_{O}+I_{O}$, and $v_{C r}$ is equal to zero at $t=t_{1}$. The negative peak value of $v_{C r}$ occurs when $i_{L r}$ is equal to $I_{O}$ at $t=t_{1} . i_{L r}$ is decreased to zero at $t=t_{2}$. The switch $Q$ is turned off automatically due to the forward direction, as shown in Figure 9. The equations of the circuit are shown as

$$
\begin{gathered}
v_{C r}\left(t_{1}\right)=V_{\mathrm{in}}, \\
i_{L r}(t)=I_{o}+\frac{V_{\text {in }}}{Z_{o}} \sin \omega_{o}\left(t-t_{1}\right)
\end{gathered}
$$

Substituting formula (3.4) into formula (3.3) and yielding

$$
v_{C r}(t)=V_{\text {in }} \cos \omega_{o}\left(t-t_{1}\right)
$$




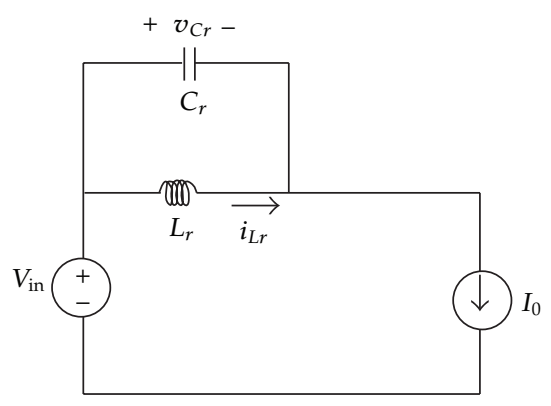

Figure 9: The equivalent circuit of Mode 2.

As a result, both $L_{r}$ and $C_{r}$ form a resonant path. From (3.4), it is necessary to have $Z_{o} I_{o}<V_{\text {in }}$ to confirm with ZCS at this moment. Mode 2 is finished at $t=t_{2}$, when the peak value of the capacitor voltage $v_{\mathrm{Cr}(\mathrm{pk})}$ is equal to $-V_{\mathrm{in}}$. The period of Mode 2 is calculated by

$$
T_{b}=\frac{\left[\sin ^{-1}\left(\left(Z_{o} I_{o}\right) / V_{\text {in }}\right)+\pi\right]}{\omega_{o}} .
$$

The pulse trigger of switch $Q$ is eliminated, and Mode 3 is entered at $t=t_{2}$.

Mode 3 [recovery stage $\left(t_{2} \leq t \leq t_{3}\right)$ ]

The equivalent circuit of Mode 3 is shown in Figure 10. $I_{o}$ passes through $C_{r}$. So $v_{C r}$ is increased linearly at this stage. The circuit equation is presented as

$$
v_{C r}(t)=\frac{I_{o}}{C_{r}}\left(t-t_{2}\right)+V_{\text {in }} \cos \omega_{o}\left(t_{2}-t_{1}\right)
$$

$v_{C r}\left(t_{3}\right)$ can be calculated by substituting $t=t_{3}$ into formula (3.7); therefore

$$
v_{C r}\left(t_{3}\right)=\frac{I_{o}}{C_{r}}\left(t_{3}-t_{2}\right)+V_{\text {in }} \cos \omega_{o}\left(t_{2}-t_{1}\right)
$$

Due to $v_{C r}\left(t_{3}\right)=V_{\text {in, }}$, the following equation can be obtained from formula (3.8):

$$
t_{3}-t_{2}=\left(\frac{V_{\text {in }} C_{r}}{I_{o}}\right)\left[1-\cos \omega_{o}\left(t_{2}-t_{1}\right)\right]
$$

Thus, the period of Mode 3 can be represented as

$$
T_{c}=\left(\frac{V_{\text {in }} C_{r}}{I_{o}}\right)\left[1-\cos \omega_{o}\left(t_{2}-t_{1}\right)\right]
$$

Mode 3 is finished at $t=t_{3}$. So $D_{m}$ is turned on at this moment, and enter to Mode 4 . 


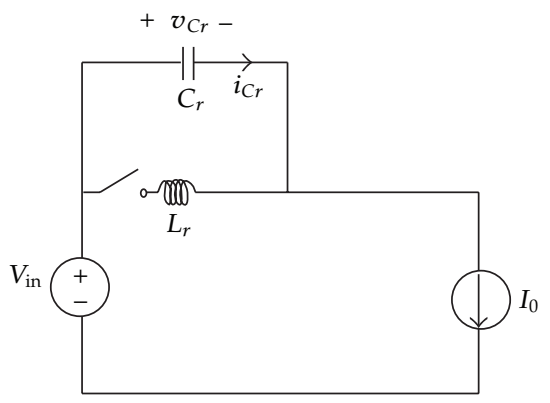

Figure 10: The equivalent circuit of Mode 3.

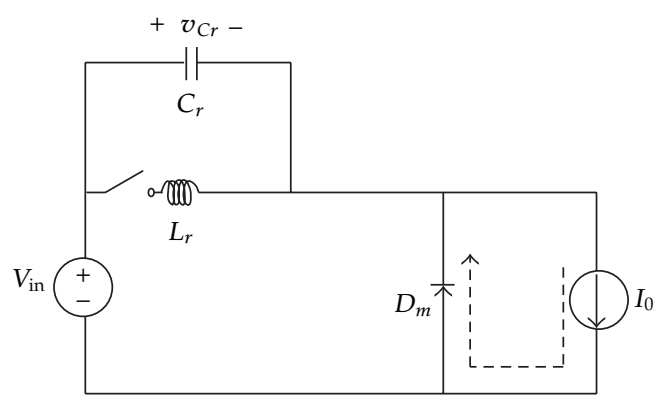

Figure 11: The equivalent circuit of Mode 4.

Mode 4 [freewheeling stage $\left(t_{3} \leq t \leq t_{4}\right)$ ]

At this stage, the switch $Q$ is still controlled under the turn-off condition. Diode $D_{m}$ is turned on and is formed of the $I_{o}$ loop, as shown in Figure 11. At $t=t_{4}$, the switch $Q$ is triggered again. Then, the next cycle begins. If we can control the period of the freewheeling stage, we can regulate the output voltage. The circuit equation is described by

$$
T_{d}=T_{s}-\left(T_{a}+T_{b}+T_{c}\right)
$$

The capacitor and inductor do not consume power in the ideal condition. In the ideal condition, no energy is wasted in switch element, transistor, or diode. Neither the capacitor nor inductor has a parasitic resistor in the ideal condition. The supply energy of the power source is equal to the absorbing energy of the load in a cycle. The circuit equation is shown as

$$
\begin{gathered}
V_{\text {in }} \int_{0}^{t_{1}} \frac{V_{\text {in }}}{L_{r}} t d t+\int_{t_{1}}^{t_{2}}\left[I_{o}+\frac{V_{\text {in }}}{Z_{o}} \sin \omega_{o}\left(t-t_{1}\right)\right] d t=\frac{V_{o} I_{o}}{f_{s}}, \\
V_{o}=V_{\text {in }} f_{s}\left\{\frac{V_{\text {in }}}{I_{o} L_{r}}\left(\frac{t_{1}^{2}}{2}\right)+\left(t_{2}-t_{1}\right)+\frac{V_{\text {in }}}{I_{o} Z_{o} \omega_{o}}\left[1-\cos \omega_{o}\left(t_{2}-t_{1}\right)\right]\right\} .
\end{gathered}
$$


After rearrangement, the average value of output voltage $V_{o}$ can be derived by

$$
V_{o}=V_{\text {in }} f_{s}\left[\left(\frac{t_{1}}{2}\right)+\left(t_{2}-t_{1}\right)+\left(t_{3}-t_{2}\right)\right] .
$$

According to above condition, formula (3.13) can be rewritten as

$$
V_{o}=V_{\mathrm{in}} f_{s}\left[\left(\frac{T_{a}}{2}\right)+T_{b}+T_{c}\right]
$$

The voltage average value of the filtering inductor is equal to zero in steady state. The average voltage of $v_{C r}$ is exactly equal to the output voltage $V_{o}$. We can modulate the value of the output voltage $V_{o}$ by controlling the period of Mode 4 (i.e., changing the switching frequency). From the waveforms of Figure 7, we can get the characteristics of the device.

(1) Both turn-on and turn-off of the switch are ZCS in order to reduce the switching loss of the switch element.

(2) The load current $I_{o}$ must be less than $V_{\text {in }} / Z_{o}$ to confirm ZCS of the switch during the turn-off period.

(3) Increasing $I_{o}$ will result in a reduced $V_{o}$ during the constant frequency operation period. We can regulate $V_{o}$ by increasing $\omega_{o}$. We can reduce $\omega_{o}$ to modulate $V_{o}$ during the $I_{o}$ decreasing period.

(4) If the switch is parallel to an antiparallel diode, the inductor current can reverse the current direction. The energy stored in the resonant circuit will be sent back to $V_{o}$.

The converter can be operated in a very high frequency region due to reduced switching loss and EMI.

\section{The Element Design of the Charger in a ZCS Quasiresonant Buck Converter}

Compared to a traditional PWM converter, the switching loss of a ZCS quasiresonant buck converter is low. This paper adopts the charger of a ZCS quasiresonant buck converter. As shown in Figure 6, a resonant capacitor $C_{r}$ and a resonant inductor $L_{r}$ were added to reduce the switching loss of switch $Q$ in the traditional PWM Buck converter circuit. According to the results of the operation stage analysis, we can design the resonant elements (i.e., resonant capacitor $C_{r}$ and resonant inductor $L_{r}$ ). According to the charger's energy balance of the ZCS quasiresonant buck converter in Figure 6, neither capacitor nor inductor consumes average energy in the ideal condition. There is no energy consumption in the switch element, transistor, or diode during the ideal condition. Thus, the supply energy of the power source is equal to the absorbing energy of the load. The supply energy of power source can be written as

$$
E_{\text {in }}=\int_{0}^{T_{S}}=P_{\text {in }}(t) d t=V_{\text {in }} \int_{0}^{T_{S}} i_{L r}(t) d t
$$




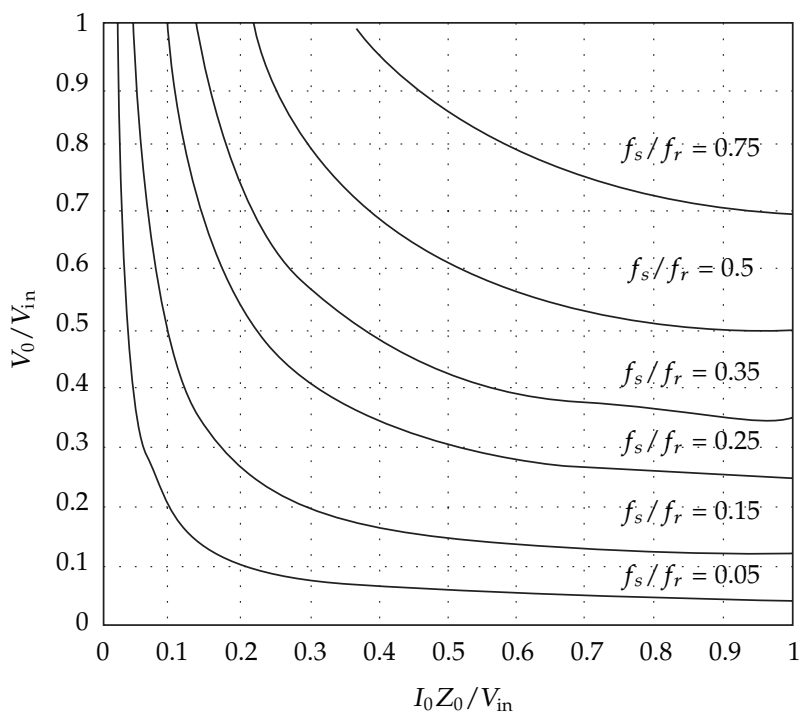

Figure 12: The load characteristics curve.

The absorbing energy of the load can be calculated by

$$
E_{o}=\int_{0}^{T_{S}} P_{o}(t) d t=V_{o} I_{o} T_{s}
$$

If we neglect the power consumption of the converter, we can assign the normalization of load resistor $r=R_{o} / Z_{o}$ and the ratio of the output voltage $X=V_{o} / V_{\text {in }}$. We can obtain formula (4.3) after simplification at $\alpha=\omega_{0}\left(t_{2}-t_{1}\right)$ :

$$
\frac{V_{o}}{V_{\mathrm{in}}}=\frac{f_{s}}{2 \pi f_{r}}\left\{\frac{X}{2 r}+\pi+\sin ^{-1}\left[\frac{X}{r}\right]+\frac{r}{X}\left[1+\sqrt{1-\left(\frac{X}{r}\right)^{2}}\right]\right\} .
$$

After converting formula (4.3) appropriately, we can get the curve sketch of the load characteristics as Figure 12 [10].

MATLAB simulation software was used to sketch the curve plot. Because the ZCS quasiresonant converter must be suitable for $Z_{o} I_{O}<V_{\text {in }}$, this paper adopts the curve for which the ratio of the output and input is the closest to $1 . f_{s} / f_{r}$ is chosen to be 0.75 . The switching frequency is equal to $22.72 \mathrm{kHz}$. The resonant frequency is $30 \mathrm{kHz}$. With $Z_{o} I_{o}<V_{\text {in }}$ $I_{o}$ is designed to equal $0.4 \mathrm{~A}$, and $V_{\text {in }}$ is equal to $24 \mathrm{~V}$. After calculating, we get $C_{r}>88.49 \mathrm{nF}$ and $L_{r}<318 \mathrm{uH}$. One adopts $C_{r}=0.1 \mathrm{uF}$ and $L_{r}=300 \mathrm{uH}$.

\section{Experiment Results and Discussion}

This study includes circuit simulations using Pspice software and practically implements the developed novel charger. Finally, the simulated and practical results are compared. The battery charger characteristics of a resonant switching converter were investigated. 


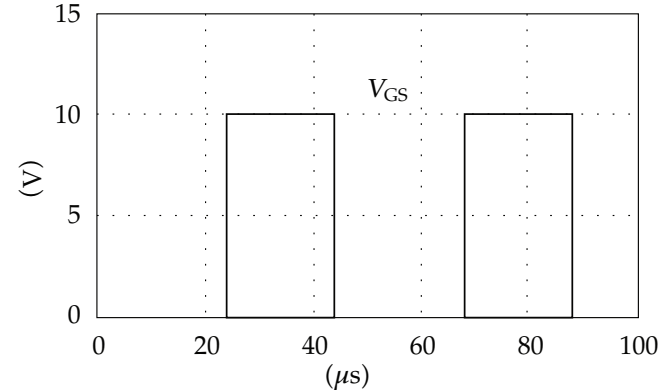

(a)

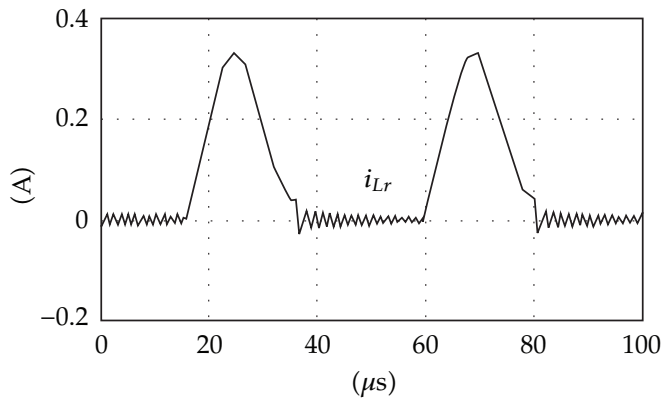

(c)

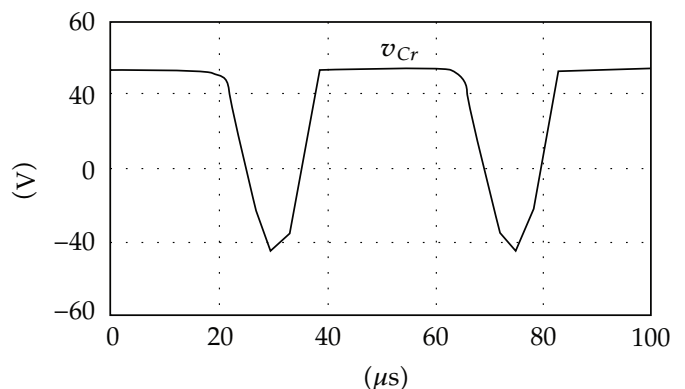

(b)

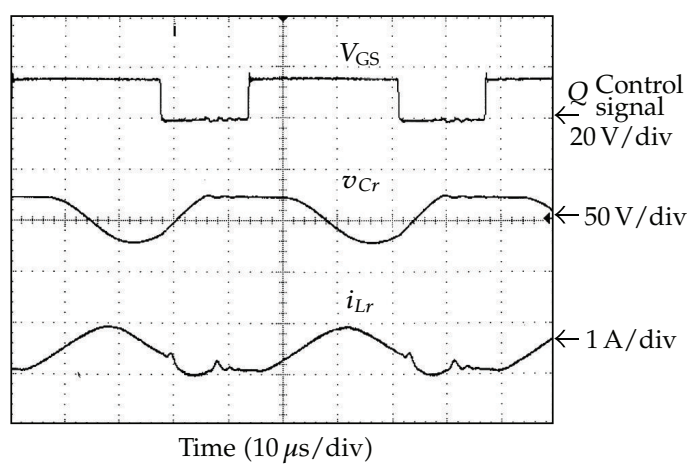

(d)

Figure 13: Waveforms of $V_{\mathrm{GS}}, v_{C r}$, and $i_{L r}$ (a) simulated waveform of $V_{\mathrm{GS}}$ (b) simulated waveform of $v_{C r}$ (c) simulated waveform of $i_{L r}(\mathrm{~d})$ practical waveform of $V_{\mathrm{GS}}, v_{\mathrm{C} r}$, and $i_{L r}$.

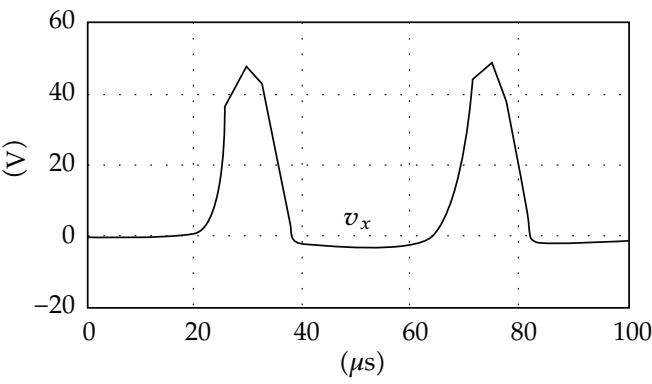

(a)

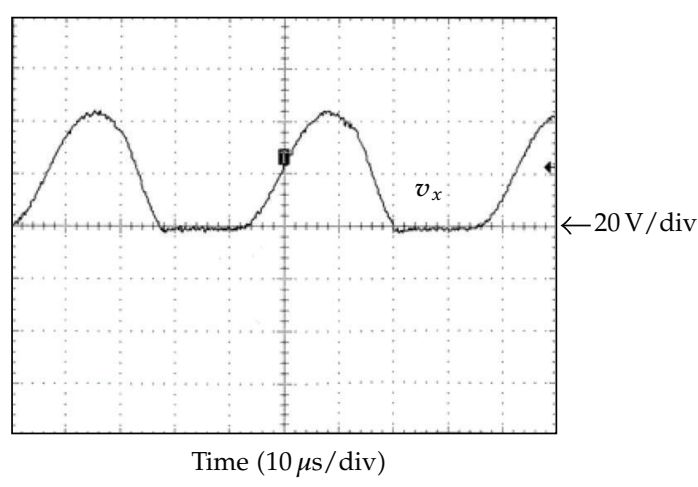

(b)

Figure 14: The voltage waveforms of diode $D_{m}$ (a) simulated waveform (b) measured waveform.

The resonant waveforms of the chargers, the curve charts of the charging periods, and the temperature curves were compared. For the experiment, the DC input voltage was $24 \mathrm{~V}$, the switching frequency of the switch was $22.72 \mathrm{kHz}$, the resonant frequency was $30 \mathrm{kHz}$, the charging current was $0.4 \mathrm{~A}$, the charging voltage was $16 \mathrm{~V}$, and the open circuit voltage was 11.7 V. Figure 13 shows the simulated and practical waveforms of the main switch triggered 


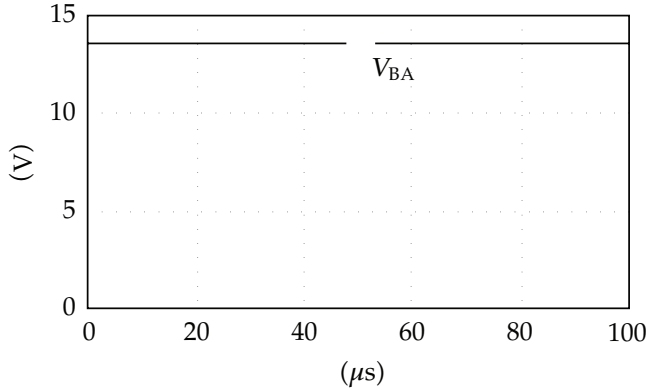

(a)

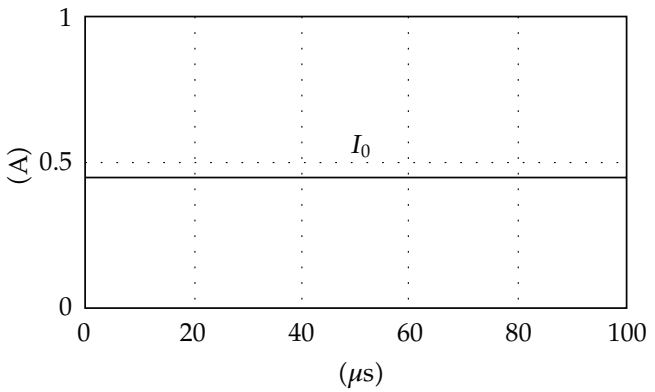

(b)

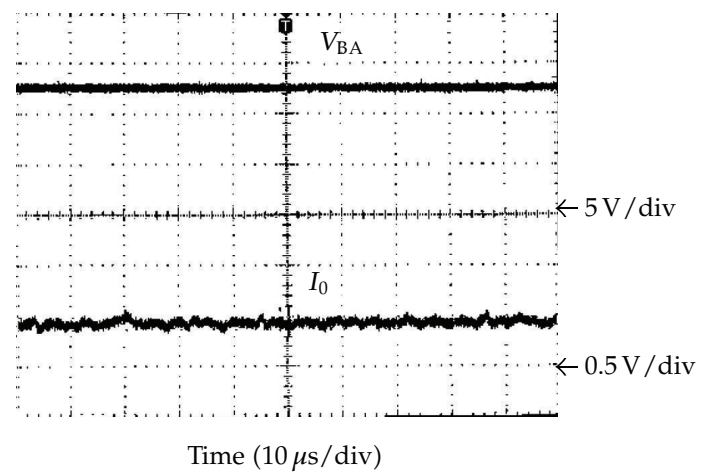

(c)

Figure 15: The waveforms of battery charging voltage and charging current (a) simulated waveform of output voltage (b) simulated waveform of output current (c) measured waveforms of charging voltage and charging current.

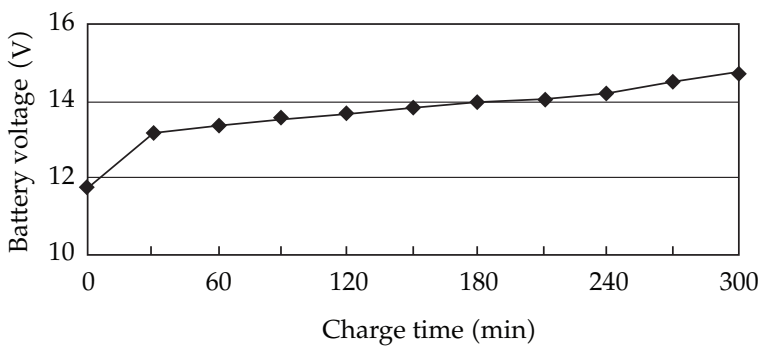

Figure 16: The elevated curves of battery voltage.

signal $V_{\mathrm{GS}}$, the resonant capacitor voltage $v_{C r}$, and the resonant inductor current $i_{L r}$ during the charging period. Compared to Figure 6, the turn-on and turn-off operation of the main switch is at the zero-current condition. The switching loss of the switch is lower than those of hard-switching ones.

Figures $14(\mathrm{a})$ and $14(\mathrm{~b})$ plot the voltage waveforms of diode $D_{m}$. The battery charging voltage and charging current are shown in Figure 15. With a $0.5 \mathrm{~A}$ constant current, it takes 180 minutes for the voltage of the lead-acid battery to reach $14 \mathrm{~V}$, as shown in Figure 16. 


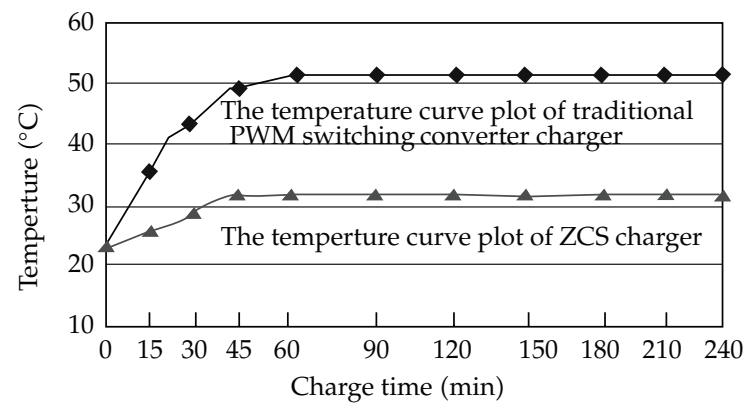

Figure 17: A comparison of power switch temperature curves.

A temperature curve comparison chart between the ZCS converter charger and traditional PWM switching ones is shown in Figure 17. A temperature comparison of the power switch is also shown in Figure 17 at the same test conditions (same output and input voltages, lead-acid battery, filtering inductor, and filtering capacitor). The charger temperature of the ZCS converter was kept at $32^{\circ} \mathrm{C}$ after a certain period. This can verify that ZVS can reduce the power loss of the power switch.

\section{Conclusion}

This paper has developed a novel application of zero-current-switching buck dc-dc converter for a battery charger. The circuit structure is simpler and much cheaper than other control mechanisms requiring large numbers of components. From the results of the experiments, charger switch is turned on and off at the zero-current stage. Resonant switching improves the traditional hard-switching power loss produced by turning the switch on and off at a nonzero current stage and lowers the switch temperature to reduce power loss of the power switch. From the measurements, the power switch transistor temperature of the ZCS converter charger was kept at $31^{\circ} \mathrm{C}$ after a certain period. Compared to a traditional hardswitching charger, the temperature of the power switch transistor of the proposed charger was much lower.

\section{References}

[1] Y. C. Chuang and Y. L. Ke, "A novel high-efficiency battery charger with a buck zero-voltageswitching resonant converter," IEEE Transactions on Energy Conversion, vol. 22, no. 4, pp. 848-854, 2007.

[2] C. Cutrona and C. di Miceli, "A unified approach to series,parallel and series-parallel resonant converters," in Proceedings of the 14th International Telecommunications Energy Conference (INTELEC'92), pp. 139-146, 1992.

[3] M. Castilla, L. G. de Vicuña, J. M. Guerrero, J. Matas, and J. Miret, "Sliding-mode control of quantum series-parallel resonant converters via input-output linearization," IEEE Transactions on Industrial Electronics, vol. 52, no. 2, pp. 566-575, 2005.

[4] M. K. Kazimierczuk, "Class D current-driven rectifiers for resonant DC/DC converter applications," IEEE Transactions on Industrial Electronics, vol. 38, no. 5, pp. 344-354, 1991.

[5] K. H. Liu and F. C. Y. Lee, "Zero-voltage switching technique in DC/DC converters," IEEE Transactions on Power Electronics, vol. 5, no. 3, pp. 293-304, 1990.

[6] M. K. Kazimierczuk, D. Czarkowski, and N. Thirunarayan, "New phase-controlled parallel resonant converter," IEEE Transactions on Industrial Electronics, vol. 40, no. 6, pp. 542-552, 1993.

[7] O. Dranga, B. Buti, and I. Nagy, "Stability analysis of a feedback-controlled resonant DC-DC converter," IEEE Transactions on Industrial Electronics, vol. 50, no. 1, pp. 141-152, 2003. 
[8] J. G. Cho, J. W. Baek, G. H. Rim, and I. Kang, "Novel zero voltage transition PWM multi-phase converters," in Proceedings of the IEEE 11th Annual Applied Power Electronics Conference and Exposition, (APEC'96), pp. 500-506, March 1996.

[9] N. H. Kutkut, H. L. N. Wiegman, D. M. Divan, and D. W. Novotny, “Design considerations for charge equalization of an electric vehicle battery system," IEEE Transactions on Industry Applications, vol. 35, no. 1, pp. 28-35, 1999.

[10] T. T. Chieng, The Beginning of Novel Soft-Switching Power Supply, Chuan Hwa Book CO., LTD, Taiwan, 2000. 


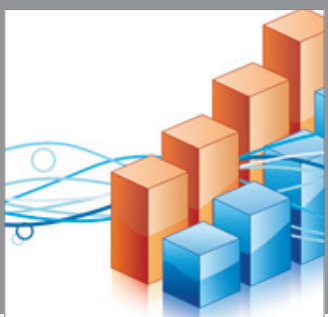

Advances in

Operations Research

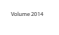

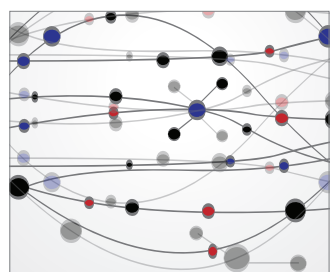

\section{The Scientific} World Journal
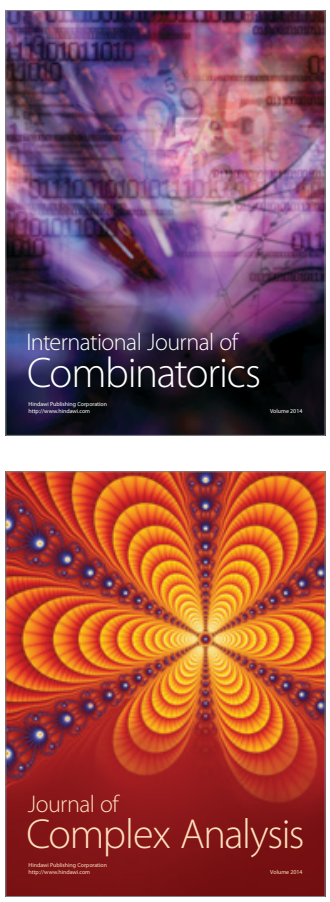

International Journal of

Mathematics and

Mathematical

Sciences
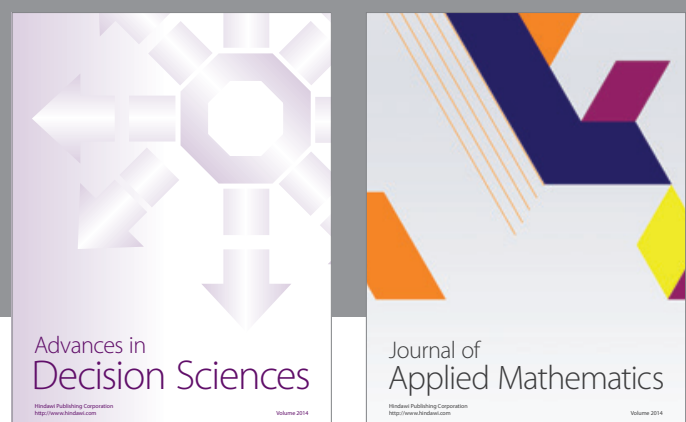

Journal of

Applied Mathematics
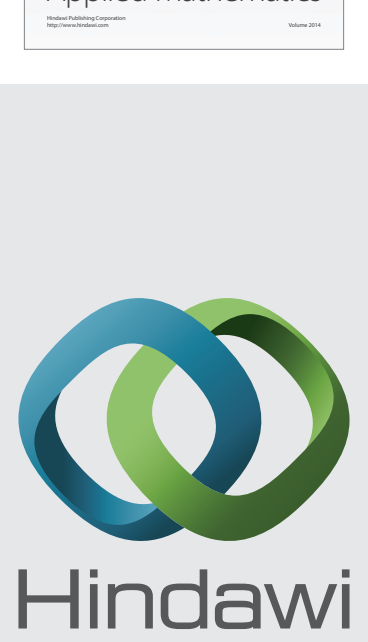

Submit your manuscripts at http://www.hindawi.com
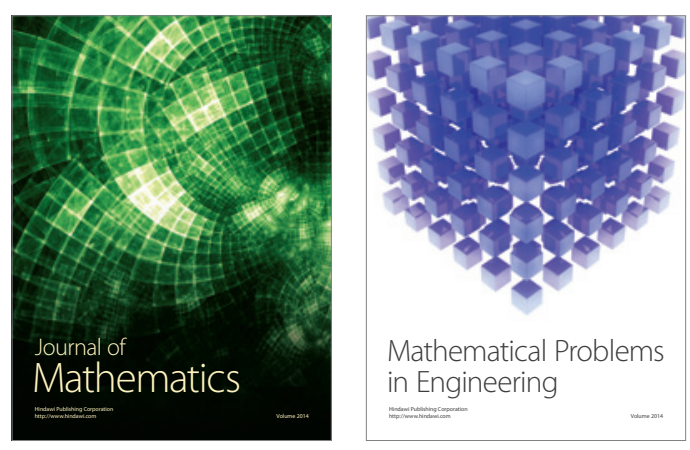

Mathematical Problems in Engineering
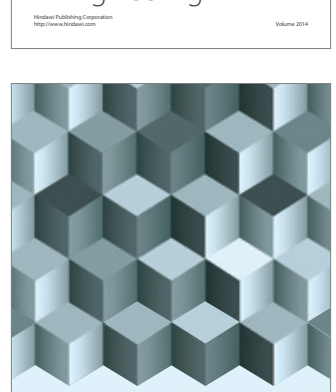

Journal of

Function Spaces
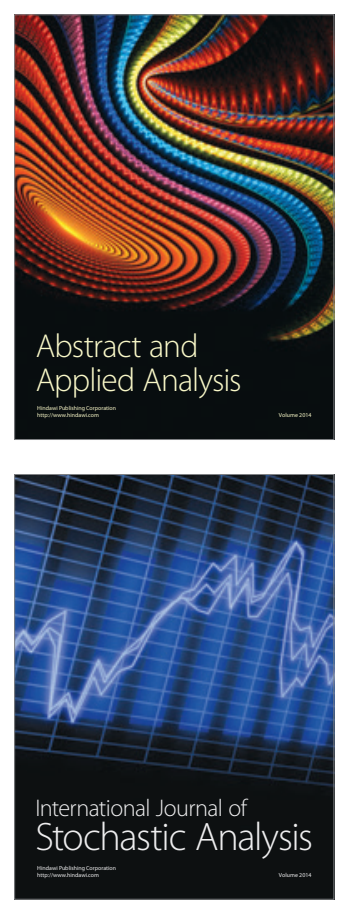

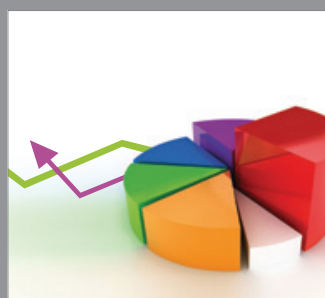

ournal of

Probability and Statistics

Promensencen
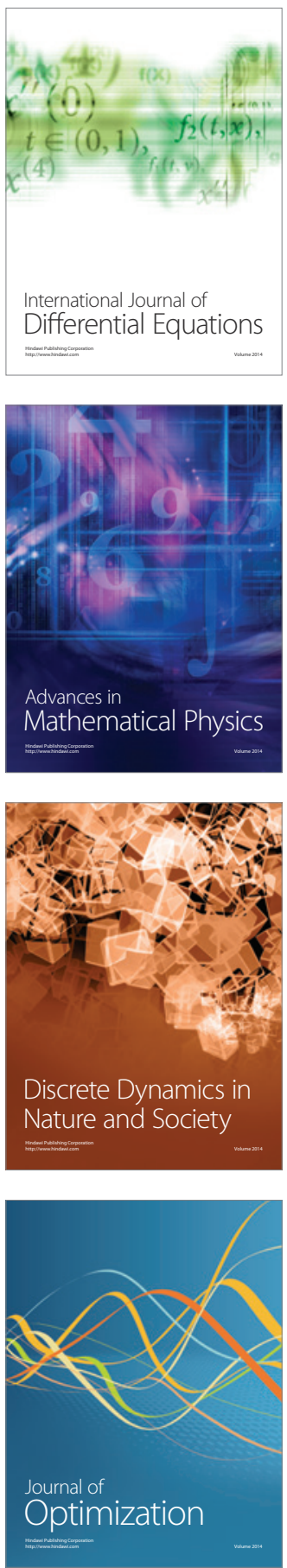